\title{
Energy-aware integrated process planning and scheduling for job shops
}

Proc IMechE Part B:

J Engineering Manufacture 2015, Vol. 229(SI) 13-26 (C) IMechE 2014

Reprints and permissions:

sagepub.co.uk/journalsPermissions.nav DOI: $10.1177 / 0954405414553069$

pib.sagepub.com

(SAGE

\author{
Min Dai ${ }^{1,2}$, Dunbing Tang ${ }^{1,2}$, Yuchun $X u^{3}$ and Weidong $\mathrm{Li}^{4}$
}

\begin{abstract}
Process planning that is based on environmental consciousness and energy-efficient scheduling currently plays a critical role in sustainable manufacturing processes. Despite their interrelationship, these two topics have often been considered to be independent of each other. It therefore would be beneficial to integrate process planning and scheduling for an integrated energy-efficient optimisation of product design and manufacturing in a sustainable manufacturing system. This article proposes an energy-aware mathematical model for job shops that integrates process planning and scheduling. First, a mixed integrated programming model with performance indicators such as energy consumption and scheduling makespan is established to describe a multi-objective optimisation problem. Because the problem is strongly nondeterministic polynomial-time hard (NP-hard), a modified genetic algorithm is adopted to explore the optimal solution (Pareto solution) between energy consumption and makespan. Finally, case studies of energy-aware integrated process planning and scheduling are performed, and the proposed algorithm is compared with other methods. The approach is shown to generate interesting results and can be used to improve the energy efficiency of sustainable manufacturing processes at the process planning and scheduling levels.
\end{abstract}

\section{Keywords}

Sustainable manufacturing, process planning and scheduling, energy consumption, makespan, genetic algorithm

Date received: 29 July 20I4; accepted: 20 August 2014

\section{Introduction}

Due to increasing environmental awareness, energy efficiency has become indispensable as a productivity criterion in manufacturing processes. Since the beginning of the industrial revolution, the industrial sector has consumed large amounts of energy for production. Manufacturing enterprises are responsible for approximately $33 \%$ of global total energy consumption and $38 \%$ of greenhouse gas emissions. ${ }^{1}$ Moreover, the need for sustainable development to attain economic, ecological and social goals is presenting new challenges to manufacturing companies. ${ }^{2}$ It is therefore important that the manufacturing community has access to systems that can improve energy efficiency in manufacturing processes by reducing energy consumption using new technologies and techniques. ${ }^{3,4}$

Process planning and scheduling are two of the most significant elements of manufacturing processes. Process planning and scheduling are usually implemented sequentially. The former is used to plan manufacturing resources (e.g. machines and tools) and operations of jobs based on cost-effective criteria, such as manufacturing cost; the latter is used to determine how and when to assign the operations of all jobs to the manufacturing resources in terms of constraints, such as time feasibility and resource availability. Due to the increasing importance of energy savings, environmental factors like energy consumption are increasingly taken into account along with traditional performance criteria (time, quality and cost) in the process planning and scheduling levels. Recently, many interesting studies have explored energy-efficient manufacturing with respect to environment-friendly process planning strategies and energy-aware scheduling approaches. Unfortunately, these two factors have been considered

\footnotetext{
'College of Mechanical and Electrical Engineering, Nanjing University of Aeronautics and Astronautics, Nanjing, China

${ }^{2}$ Jiangsu Key Laboratory of Precision and Micro-Manufacturing Technology, Nanjing, China

${ }^{3}$ School of Applied Sciences, Cranfield University, Cranfield, UK

${ }^{4}$ Faculty of Engineering and Computing, Coventry University, Coventry, UK
}

\section{Corresponding author:}

Dunbing Tang, College of Mechanical and Electrical Engineering, Nanjing University of Aeronautics and Astronautics, Nanjing 210016 , China.

Email: d.tang@nuaa.edu.cn 
independently. As a result, energy consumption in process planning systems has not obtained optimal energy savings due to resource constraints in the scheduling systems. In addition, energy-efficient scheduling with regard to energy-saving information, which is generated after process planning strategies are implemented, cannot be fed back to the process planning system to improve it. It is therefore necessary to integrate these factors to enhance energy-efficient manufacturing.

The following sections of this article are organised as follows. Related studies are introduced in section 'Related work'. An energy-aware integrated process planning and scheduling (EIPPS) problem is addressed in section 'EIPPS'. A modified heuristic algorithm optimisation approach for the EIPPS problem is described in section 'A modified genetic algorithm optimisation approach'. Case studies are presented in section 'Case studies', and the conclusions are summarised in section 'Conclusion and future research'.

\section{Related work}

\section{Energy-efficient process planning}

Process planning describes the transformation of raw materials into products through planning the operations of a product based on machining features, the identification of manufacturing resources that are available to the operations and the determination of the machining sequence in terms of cost-effective indicators. It has a direct influence on the design and manufacturing of products, which are closely related to environmental impacts such as energy consumption. Many studies of energy-efficient process planning have been performed. Sheng and Srinivasan ${ }^{5}$ presented an environmentally conscious multi-objective process planning approach that is based on manufacturing features and explored local and global optimum process planning with respect to process energy consumption, process time, waste mass and surface quality factors at the micro- and macro-planning levels. ${ }^{6,7}$ Singh et al. ${ }^{8}$ developed a framework and models of environmental process planning to assess configurations of a product and its related environmental impact during the advanced product quality planning (APQP) process. Kai et al. ${ }^{9}$ developed a multi-objective mathematical model for environmentally supportive process planning that simultaneously considers cost, time and environmental impact. Newman et al. ${ }^{10}$ introduced energy consumption as an objective of process planning for computer numerical control (CNC) machining and confirmed that energy consumption can be used as a performance indicator in a multi-criteria process planning system. Yin et al. ${ }^{11}$ proposed a new process planning approach that considers environmental factors, such as carbon emissions, and obtained a comparatively green and economical process plan. In summary, a process plan provides a framework to make energy-efficient decisions that minimise energy consumption while maintaining expected production objectives such as time, cost and quality.

\section{Energy-efficient scheduling}

Scheduling generally involves planning how and when to assign the operations of all jobs to manufacturing resources based on the priority of the jobs, the availability of machines and tools, and time constraints. When scheduling takes into account environmental impacts like energy consumption as an optimisation objective, it is energy efficient. Recently, research on minimising energy consumption in manufacturing processes through scheduling has been gradually increasing. One of the best-known studies of the impact of scheduling on energy efficiency was Mouzon et al., ${ }^{12}$ who proposed a multi-objective mathematical programming model and several algorithms to investigate the scheduling of jobs on a single CNC machine with the goals of reducing energy consumption and total completion time. Mouzon and Yildirim ${ }^{13}$ outlined a multiobjective optimisation schedule with the objective of minimising the total energy consumption and the total tardiness on a machine using a greedy randomised adaptive search algorithm. Fang et al. ${ }^{14}$ provided a new mixed integer linear programming model to schedule a classical flow shop that incorporated the peak total power consumption, the carbon footprint and the makespan. Bruzzone et al. ${ }^{15}$ reported an energy-aware scheduling algorithm that is based on a mixed integer programming formulation to realise energy savings for a flexible flow shop that was required to maintain the original job assignment and sequencing. Zhang et al. ${ }^{16}$ developed a mathematical model to minimise energy consumption and improve scheduling efficiency for the dynamic scheduling problem in a flexible manufacturing system. Liu et al. ${ }^{17}$ considered reducing the total wasted energy consumption using a branch and bound algorithm in a permutation flow shop scheduling problem. Dai et al. ${ }^{18}$ also explored the multi-objective energy-efficient scheduling problem with the makespan and energy consumption of manufacturing processes as objectives. The energy-aware schedules in these studies were developed after process planning generating the operations of all jobs. Because the assumption that all of the manufacturing resources will be available at the process planning level might not be valid at the scheduling level, the scheduling results might not be optimised.

\section{Integrated process planning and scheduling}

Process planning and scheduling play critical roles in linking product design and manufacturing and must be integrated on the basis of their complementary relationship in manufacturing processes. The preliminary concept of integrated process planning and scheduling (IPPS) was first proposed by Chryssolouris et al. ${ }^{19,20}$ Numerous studies by several researchers have subsequently explored IPPS, and three main models for 
IPPS have been developed: non-linear process planning, closed-loop process planning and distributed process planning. The research objectives for IPPS mostly focus on traditional production performance indicators, such as the makespan, the job tardiness, the balanced level of machine utilisation and the manufacturing cost. ${ }^{21-26}$ However, most of these process planning and scheduling methods have paid little attention to the energy efficiency of manufacturing processes. This study proposes an energy-aware mathematical model for IPPS to achieve energy-saving design and manufacturing of a product based on a non-linear process planning method. The proposed model simultaneously generates the energy-efficient process planning strategy of all jobs and the energy-efficient scheduling strategy. The EIPPS strategies are deployed by the multi-objective optimisation function with two objectives: the energy consumption and the makespan on the job-shop floor. In addition, a modified genetic algorithm (GA) is adopted to determine the optimal solution of the model.

\section{EIPPS}

\section{Problem description}

According to the definition of the IPPS problem, ${ }^{24}$ the EIPPS formulation can be described as follows. A set of $n$ jobs $J=\{1,2, \ldots, n\}$ are to be processed on a set of $m$ machines $M=\{1,2, \ldots, m\}$. Each job $j \in J$ is characterised by a set of $o_{l j}\left(l \in G_{j}, j \in J\right)$ operations $O_{l j}=\{1$, $\left.2, \ldots, o_{l j}\right\}$, including a set of $g_{j}$ alternative process plans $G_{j}=\left\{1,2, \ldots, g_{j}\right\}$ that have to be executed by selecting a desirable process plan. The operations of all of the jobs are to be assigned to available manufacturing resources, such as machines and tools, to determine a schedule based on several constraints among the operations. Furthermore, one job operation in different process plans could be processed on different machines with different power (energy) consumptions or on the same machine with different processing parameters, such as cutting speed. Thus, the operations of each job $j \in J$ on machine $i \in M$ have processing times and corresponding energy consumptions. The objective of the EIPPS is to assign jobs to machines and to determine the processing sequence of operations on each machine to search for optimal or near-optimal results (the Pareto solution) between the energy consumption and the maximum completion time, that is, makespan. The connection between the process planning and scheduling functions is maintained through the operations of the jobs, which is illustrated in Figure 1.

The EIPPS should satisfy the following constraints:

1. All of the jobs and machines are available at time zero.

2. Each machine should not process more than one job at a time.
3. The different operations of each job must be processed sequentially on machines.

4. Pre-emption is not allowed while executing an operation on a machine, that is, once one operation has started, it must be finished without interruption.

5. There are no precedence relationships between operations of different jobs, but there are precedence relationships between different operations of one job.

6. The power (energy) consumption can be calculated for each job that is processed on each machine.

\section{Mathematical model}

The energy-aware modelling of the IPPS problem is defined in this section. This article considers a scheduling problem that arises on a job-shop floor. The mixed integer programming model is constructed to minimise the energy consumption in the development of process planning and scheduling while satisfying the possible optimisation of the makespan. The notations used to describe the model are as follows:

- $i, i^{\prime}$ are the indexes for the position of the process on the machine;

- $\quad l, r$ are the indexes for the process plan;

- $j, p$ are the indexes for the job;

- $k, k^{\prime}, q$ are the indexes for the operation;

- $m, m^{\prime}$ are the indexes for the machine;

- $J$ is the set of jobs, $J=\{1,2, \ldots, n\}$;

- $M$ is the set of machines, $M=\{1,2, \ldots, m\}$;

- $O_{l j}$ is the set of operations of the alternative process plan $l$ of job $j, O_{l j}=\left\{1,2, \ldots, o_{l j}\right\}$, where $o_{l j}$ is the maximum number of operations of the alternative process plan $l$ of job $j$;

- $G_{j}$ is the set of alternative process plans of job $j$, $G_{j}=\left\{1,2, \ldots, g_{j}\right\}$, where $g_{j}$ is the maximum number of alternative process plans of job $j$;

- $\quad P_{m}$ is the set of operations processed on machine $m$, $P_{m}=\left\{1,2, \ldots, p_{m}\right\}$, where $p_{m}$ is the maximum number of operations processed on machine $m$;

- $\mathrm{T}_{k l j}^{m}\left(k \in O_{l j}, l \in G_{j}, j \in J, m \in M\right)$ is the processing time of operation $k$ in the $l$ th alternative process plan of job $j$ on machine $m$;

- $S_{k l j}^{i m}\left(i \in P_{m}, k \in O_{l j}, l \in G_{j}, j \in J, m \in M\right)$ is the start time of operation $k$, which is the $i$ th position processed on machine $m$, in the $l$ th alternative process plan of job $j$;

- $C_{k l j}^{i m}\left(i \in P_{m}, k \in O_{l j}, l \in G_{j}, j \in J, m \in M\right)$ is the completion time of operation $k$, which is the $i$ th position processed on machine $m$, in the $l$ th alternative process plan of job $j$;

- $C_{\max }$ is the completion time of the last operation in the schedule, that is, the makespan of the schedule;

- $\operatorname{Pc}_{k l j}^{m}\left(k \in O_{l j}, l \in G_{j}, j \in J, m \in M\right)$ is the cutting power consumption of the $k$ th operation, which belongs to the $l$ th alternative process plan of job $j$ processed on machine $m$; 


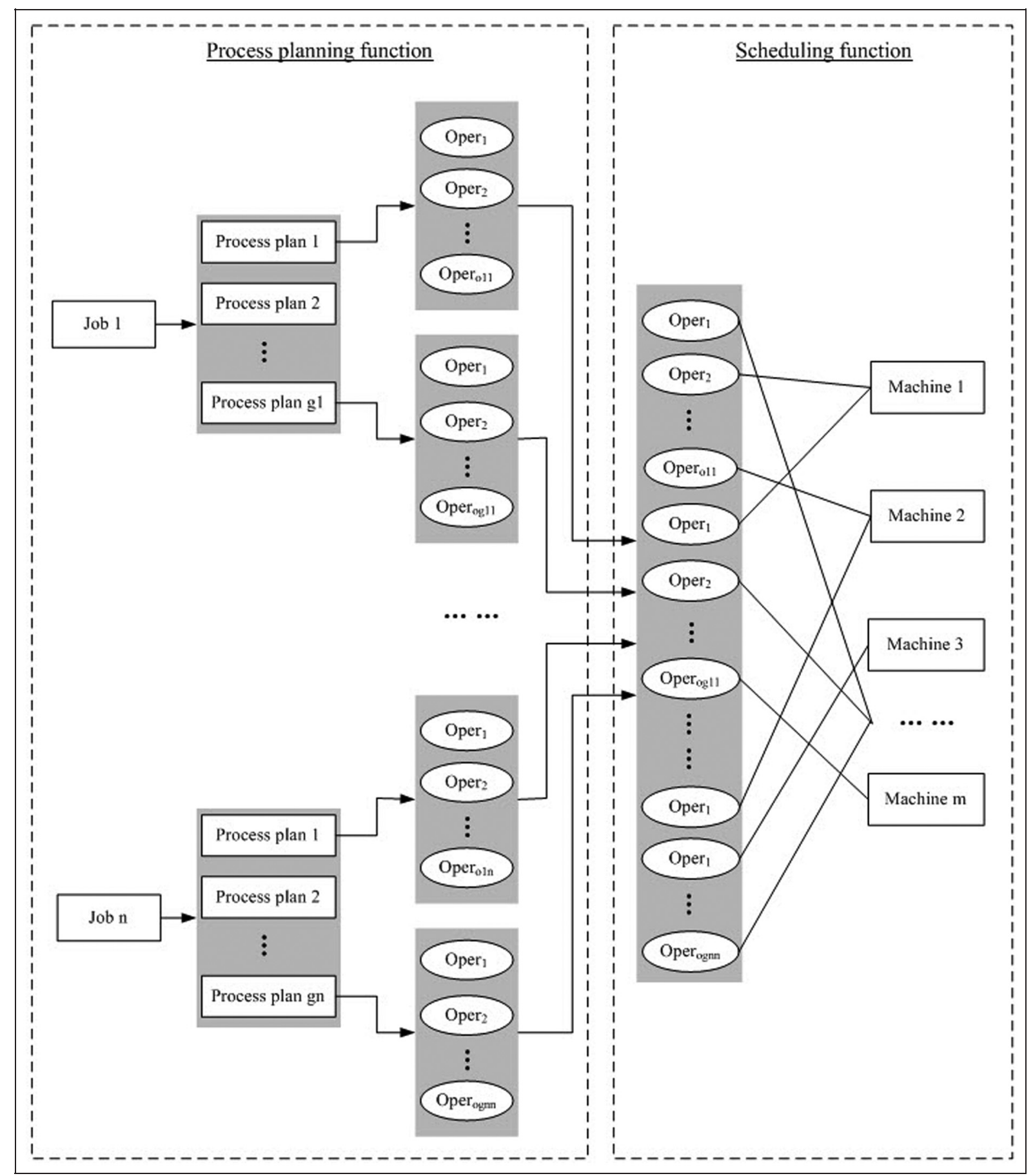

Figure I. Interactions of process planning and scheduling.

- $\operatorname{Pu}_{k l j}^{m}\left(k \in O_{l j}, l \in G_{j}, j \in J, m \in M\right)$ is the unload power consumption of the $k$ th operation, which belongs to the $l$ th alternative process plan of job $j$ processed on machine $m$;

- $\quad L$ is a very large positive number;

- $X_{l j}\left(l \in G_{j}, j \in J\right)$ is equal to 1 if the $l$ th alternative process plan is picked for job $j$ and 0 otherwise;

- $Y_{k l i}^{i m}\left(i \in P_{m}, k \in O_{l j}, l \in G_{j}, j \in J, m \in M\right)$ is equal to 1 if operation $k$ in the $l$ th alternative process plan of job $j$ is the $i$ th position processed on machine $m$ and 0 otherwise;
- $Z_{k l j q r p}^{m}\left(k, q \in O_{l j, r p}, l, r \in G_{j, p}, j, p \in J, m \in M\right) \quad$ is equal to 1 if operation $k$ in the $l$ th alternative process plan of job $j$ precedes operation $q$ in the $r$ th alternative process plan of job $p$ on machine $m$ and 0 otherwise.

The following multi-objective mixed integer programming mathematical model (1) minimises the total energy consumption $\left(f_{1}\right)$ and (2) minimises the make$\operatorname{span}\left(f_{2}\right)$. 
Minimising the total energy consumption. The energy consumption model is based on existing research on energy-efficient manufacturing processes. ${ }^{18}$ The total power consumption of manufacturing processes can be divided into three types: basic power consumption, unload power consumption and cutting power consumption. Basic power is used to maintain the normal operation of machine components, including the power consumption of the motor drive components, main spindle drive components, servo feed drive components and auxiliary components, such as hydraulic, cooling and lubrication, control and periphery components. Unload power corresponds to activities such as the loading, unloading, positioning and clamping of the workpiece as well as changing cutting tools. Cutting power corresponds to the actual cutting operation. For the energy-efficient manufacturing processes considered in this article, the primary contributors to the total energy consumption are the unload power consumption and the cutting power consumption. Under this assumption, the objective of minimising the total energy consumption, which consists of the direct energy consumed by removing material in productive modes and the indirect energy usage in non-productive modes, such as the standby stage, can be expressed as

$$
\begin{aligned}
f_{1} & =\min \sum_{k \in O_{l j}} \sum_{l \in G_{j}} \sum_{j \in J} \sum_{i \in P_{m}} \sum_{m \in M} \\
& \left(\left(\alpha \cdot\left(\mathrm{Pc}_{k l j}^{m}\right)^{2}+\beta \cdot \mathrm{Pc}_{k l j}^{m}+\mathrm{Pu}_{k l j}^{m}\right) \cdot \mathrm{T}_{k l j}^{m} \cdot X_{l j} \cdot Y_{k l j}^{i m}\right) \\
& +\sum_{k, q \in O_{l j, r p}} \sum_{l, r \in G_{j, p}} \sum_{j, p \in J} \sum_{i \in P_{m}} \sum_{m \in M} \\
& \left(\mathrm { Pu } _ { k l j } ^ { m } \cdot \left(\left(C_{k l j}^{i m}-\mathrm{T}_{k l j}^{m}\right) \cdot X_{l j} \cdot Y_{k l j}^{i m}\right.\right. \\
& \left.\left.-C_{q r p}^{(i-1) m} \cdot X_{r p} \cdot Y_{q r p}^{(i-1) m}\right) \cdot X_{l j} \cdot X_{r p} \cdot Z_{k l j q r p}^{m}\right)
\end{aligned}
$$

where the first part on the right side of the equation is the direct energy consumption of removing material in the productive stage, $\alpha, \beta$ are the coefficients of the load power consumption, which can be obtained from the linear regression equations based on the idle power consumption at the different spindle speeds, ${ }^{27}$ and the second part on the right side of the equation is the indirect energy consumption, such as the energy for standby.

Minimising the makespan. The objective of minimising the makespan, which is the maximum completion time of all jobs, can be defined as

$$
f_{2}=\min \max _{j \in J}\left(C_{k l j}^{i m} \cdot X_{l j} \cdot Y_{k l j}^{i m}\right)
$$

The objectives are subjected to the following constraints:

1. For the last operation in the $l$ th alternative process plan of job $j$ on machine $m$

$$
\begin{aligned}
& C_{\max } \geqslant\left(C_{k l j}^{p_{m} m} \cdot X_{l j} \cdot Y_{k l j}^{p_{m} m}\right)-L\left(1-X_{l j}\right), \\
& k \in O_{l j}, l \in G_{j}, j \in J, m \in M
\end{aligned}
$$

2. The earliest completion time of one operation is

$$
\begin{aligned}
& C_{k l j}^{i m} \cdot X_{l j} \cdot Y_{k l j}^{i m}=S_{k l j}^{i m} \cdot X_{l j} \cdot Y_{k l j}^{i m}+\mathrm{T}_{k l j}^{m} \cdot X_{l j} \cdot Y_{k l j}^{i m}, \\
& i \in P_{m}, k \in O_{l j}, l \in G_{j}, j \in J, m \in M
\end{aligned}
$$

3. One machine can only process one job at a time

$$
\begin{aligned}
& S_{q r p}^{i m} \cdot X_{r p} \cdot Y_{q r p}^{i m}+L \cdot\left(1-X_{l j} \cdot X_{r p} \cdot Y_{q r p}^{i m} \cdot Y_{k l j}^{(i-1) m} \cdot Z_{k l j q r p}^{m}\right) \\
& \geqslant \mathrm{T}_{k l j}^{m} \cdot X_{l j} \cdot Y_{k l j}^{(i-1) m}+S_{k l j}^{i m} \cdot X_{l j} \cdot Y_{k l j}^{(i-1) m}, \\
& i \in P_{m}: i>2, k, q \in O_{l j, r p}, l, r \in G_{j, p}, j, p \in J, m \in M
\end{aligned}
$$

4. Different operations of the job cannot be performed at the same time

$$
\begin{aligned}
& S_{k l j}^{i m} \cdot X_{l j} \cdot Y_{k l j}^{i m}-S_{k^{\prime} l j}^{i^{\prime} m^{\prime}} \cdot X_{l j} \cdot Y_{k^{\prime} l j}^{i^{\prime} m^{\prime}} \\
& +L \cdot\left(1-X_{l j}\right) \geqslant \mathrm{T}_{k^{\prime} l j}^{m^{\prime}} \cdot X_{l j} \cdot Y_{k^{\prime} l j}^{i^{\prime} m^{\prime}}, \\
& i, i^{\prime} \in P_{m}, k, k^{\prime} \in O_{l j}: k>k^{\prime}, \\
& l \in G_{j}, j \in J, m, m^{\prime} \in M
\end{aligned}
$$

5. Each job can select only one alternative process plan

$$
\sum_{l \in G_{j}} X_{l j}=1, j \in J
$$

6. Each operation can select only one machine

$$
\sum_{m \in M} Y_{k l j}^{i m}=1, i \in P_{m}, k \in O_{l j}, l \in G_{j}, j \in J
$$

7. The time should be non-negative

$$
C_{k l j}^{i m} \cdot X_{l j} \cdot Y_{k l j}^{i m} \geqslant 0, i \in P_{m}, k \in O_{l j}, l \in G_{j}, j \in J, m \in M
$$

Constraint (3) ensures that the completion time of the operation, which is the last position processed on one machine, is not greater than the makespan of the schedule. Constraint (4) notes that an operation cannot pre-empt another on a machine. Constraint (5) is the machine constraint; it allows each machine to process a maximum of one operation at once. Constraint (6) represents the operational constraints and prevents different operations for a job from being executed simultaneously. Constraint (7) states that only one alternative process plan can be selected for each job. Constraint (8) ensures that each operation will be processed by only one machine tool at a time; in other words, it does not allow an operation to be executed on more than one machine at any time. Constraint (9) ensures that the completion time of each operation will be non-negative. 


\section{A modified GA optimisation approach}

For a job-shop floor with various resources available to the operations of all given jobs, there is an enormous search space for determining the energy-aware modelling of the IPPS problem. The mixed integer programming formula for the EIPPS problem described above is a multi-objective function with constraints. Because process planning and scheduling individually are NPhard, their integration is also NP-hard. ${ }^{28}$ Hence, it is imperative to explore optimal or near-optimal solutions based on intelligent algorithms to facilitate the search and optimisation process according to the IPPS criteria. In this study, a modified GA that combines a GA with a simulated annealing algorithm (SAA) is adopted to search for the optimal solutions of the objective functions discussed above. A GA is a searching technique that is based on the process of natural evolution. ${ }^{29}$ GAs have been widely applied to combinational and other optimisation problems, such as scheduling problems. One of the remarkable advantages of GAs is being able to quickly and efficiently obtain a good solution for an objective function in a complex solution space, but a major disadvantage is potentially being trapped in a local optimum, which is called premature convergence. An SAA is a local searching technique that is analogous to annealing in solids. One of the most famous studies of SAAs is Kirkpatrick et al., ${ }^{30}$ who successfully applied an SAA to combinational optimisation problems like travelling salesman problems. Two of its prominent advantages are avoiding convergence to a local optimum and efficiently determining the global optimum of an objective function in a complex solution space. Therefore, this article proposes to incorporate the strengths of a SAA into a GA. The modified GA for the EIPPS problem is illustrated in Figure 2.

\section{Representation}

Each chromosome that is based on the multi-layer encoding scheme in process planning and scheduling is composed of two layer strings as shown in Figure 3. One represents an alternative process plan gene-string, and the other represents a scheduling plan gene-string. In the first layer encoding, the $h$ th position denotes job $h$, and the element of each position corresponds to a selected process plan of the job. For example, the fourth position of the process plan gene-string represents job 4 , and job 4 picks the first alternative process plan (Figure 3). The gene element of the fourth position is equal to 1 . Therefore, the length of the alternative process plan gene-string is dependent on the number of jobs. In the second layer encoding, the encoding scheme is based on an operation representation, which means that different appearances of the same job number denote different operations of the job, and the $p$ th appearance represents the $p$ th operation of the job. Specifically, there are five different appearances for job 4 in the scheduling plan gene-string, which means that

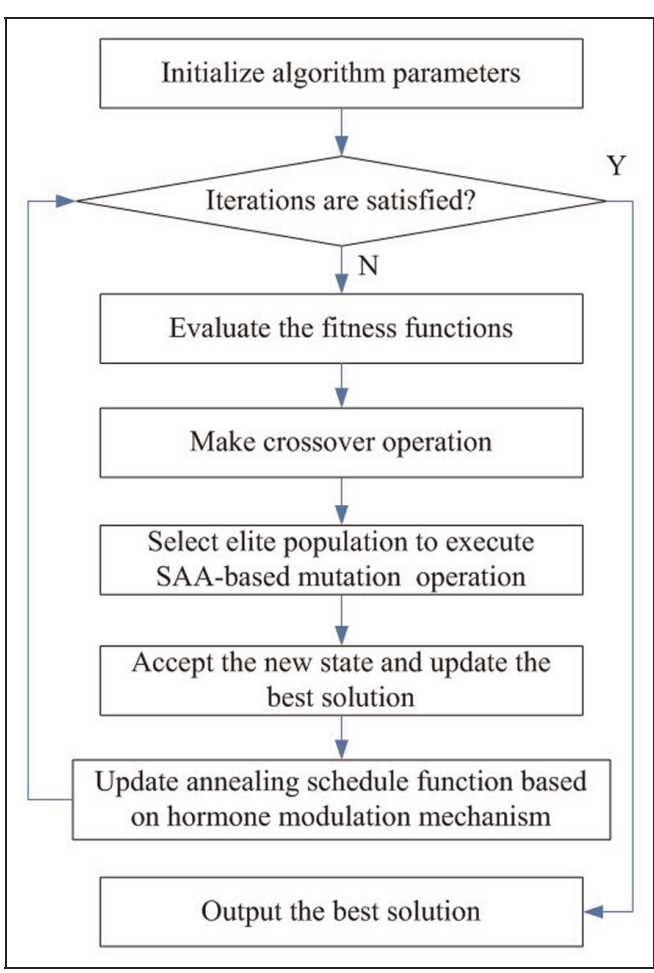

Figure 2. Flow chart of the modified genetic algorithm. SAA: simulated annealing algorithm.

job 4 has five operations. The first appearance denotes the first operation of job 4 , the second denotes the second operation of job 4 and so on. In addition, the length of each alternative process plan for a job could be different. To generate a feasible initial population, the length of each chromosome is set as follows. First, the length of an alternative process plan gene-string is set as the total number of jobs. Second, the number of operations $\left(o_{l j}\left(l \in G_{j}, j \in J\right)\right)$ of job $j$, which has the maximum operations among $g_{j}(j \in J)$ alternative process plans, is expressed as $\max _{l}\left(o_{l j}\right)$. The total length of the scheduling plan gene-string is thus equal to the sum of the maximum length of each job, that is, $\sum_{j=1}^{n} \max _{l}\left(o_{l j}\right)$. Hence, the total length of the chromosome is equal to $n+\sum_{j=1}^{n} \max _{l}\left(o_{l j}\right)$. If the length of the selected process plan gene-string of job $j$ does not correspond to the maximum number $\left(\max _{l}\left(o_{l j}\right)\right)$ in the decoding process, the elements of the operations of job $j$ are removed from the last operation position to the first until the length is satisfied by the selected position. Figure 3 shows six jobs, and the maximum number of operations of each job is 5. Thus, the total length of the chromosome is 36. Assume that the first process plan of job 4 has only four operations, which means that the last operation of job 4 in the chromosome is to be removed in the decoding process.

\section{Fitness evaluation}

In this study, we consider two objectives to find a set of efficient results in a solution space: 


\begin{tabular}{|c|c|c|c|c|c|c|c|c|c|c|c|c|c|c|c|c|c|c|c|c|c|c|c|c|c|c|c|c|c|c|c|c|c|}
\hline \multicolumn{34}{|c|}{ process plan gene-string } \\
\hline 3 & 1 & 1 & 1 & 2 & 2 & 6 & 6 & 4 & 3 & 5 & 2 & 2 & 1 & 4 & 3 & 1 & 4 & 6 & 2 & 3 & 4 & 1 & 15 & 4 & 6 & 2 & 5 & \begin{tabular}{l|l}
5 & 5
\end{tabular} & $5 \quad 2$ & 3 & 6 & 3 & 1 \\
\hline
\end{tabular}

Figure 3. Chromosome of the process plan and scheduling.

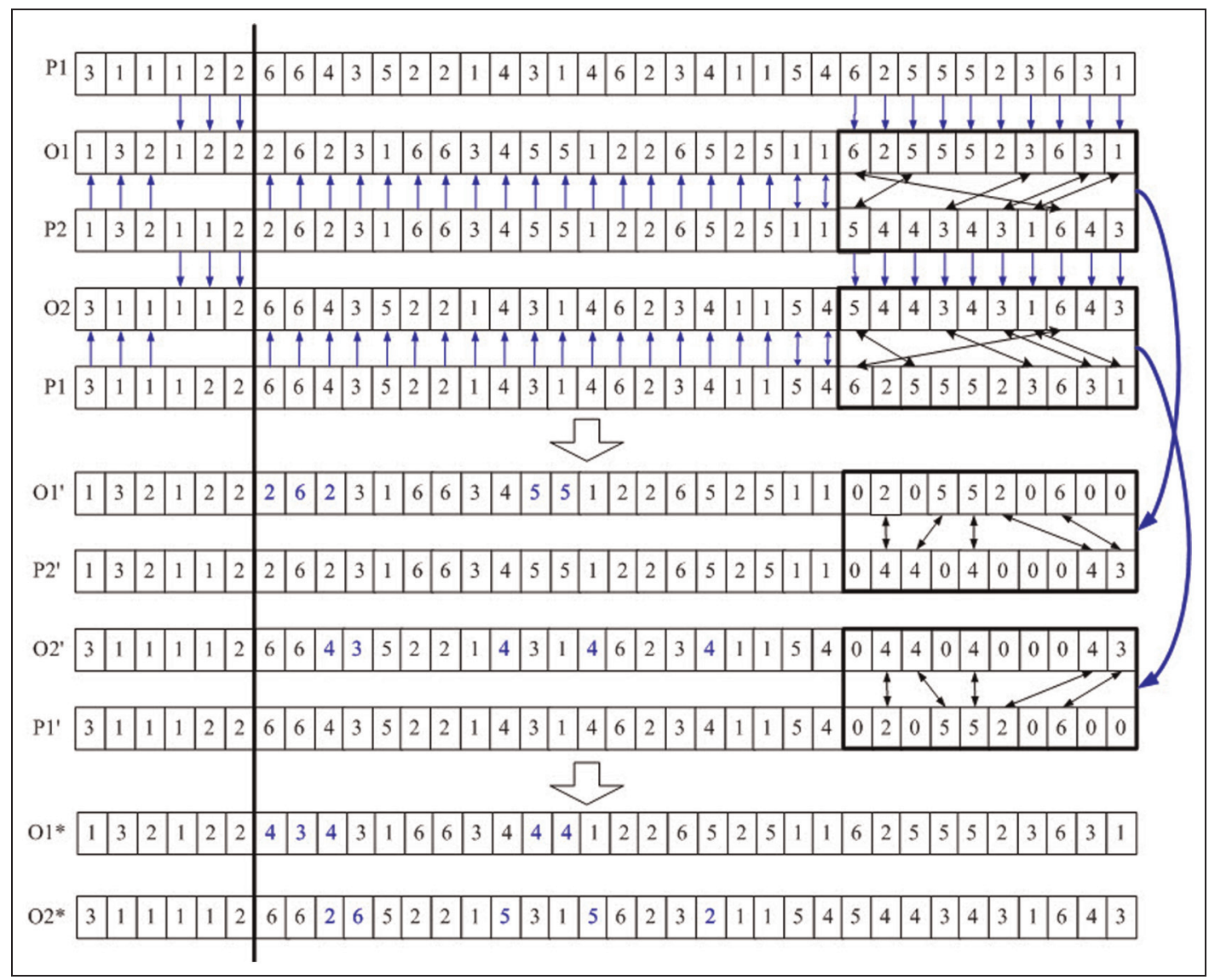

Figure 4. Crossover for a pair of chromosomes of process plan and scheduling.

1. $f_{1}$ : Minimise the total energy consumption.

2. $f_{2}$ : Minimise the makespan.

\section{Genetic operators}

Traditional GAs generally consist of three genetic operators, the selection, crossover and mutation operators, which play a significant role in the performance of the algorithm. The selection operator is responsible for picking elite chromosomes from the current population to generate the next population, which is used for the crossover and mutation operations. The crossover operator is the main way to produce new chromosomes with the parents' information, and the mutation operator is used to create new individuals with new information.

Selection operator. In this study, the rank-based selection approach is employed to produce excellent individuals for the next generation. In the rank-based selection mechanism, the best individuals are chosen with the desired probability from the parents and offspring chromosomes. In this way, an elite population can be obtained because the mechanism will only accept improvements.

Crossover operator. The procedure of the crossover operation is designed as follows, and a crossover instance is shown in Figure 4.

Step 1. Choose two parent individuals P1 and P2 at random and construct two empty offspring individuals $\mathrm{O} 1$ and $\mathrm{O} 2$.

Step 2. Generate the alternative process plan genestrings of $\mathrm{O} 1$ and $\mathrm{O} 2$.

Step 2.1. Select a crossover point for a pair of process plan gene-strings at random. Each process plan gene-string can be separated into right and left parts from the crossover point. 
Step 2.2. Copy the genes that are in the right parts of the alternative process plan gene-strings of P1 and P2 based on the position of the crossover point into the same positions as the offspring individuals $\mathrm{O} 1$ and $\mathrm{O} 2$, respectively.

Step 2.3. Copy the genes that are in the left parts of the alternative process plan gene-strings of P1 and P2 based on the position of the crossover point into the same positions as the offspring individuals $\mathrm{O} 2$ and $\mathrm{O} 1$, respectively.

Step 3. Generate the scheduling plan gene-strings of $\mathrm{O} 1$ and $\mathrm{O} 2$.

Step 3.1. Select a crossover point for a pair of scheduling plan gene-strings at random.

Step 3.2. Copy the genes that are in the right parts of the scheduling plan gene-strings of P1 and P2 based on the position of the crossover point into the same positions as the offspring individuals $\mathrm{O} 1$ and $\mathrm{O} 2$, respectively.

Step 3.3. Copy the genes that are in the left parts of the scheduling plan gene-strings of P1 and P2 based on the position of the crossover point into the same positions as the offspring individuals $\mathrm{O} 2$ and $\mathrm{O} 1$, respectively.

Step 3.4. Consider the right parts of the scheduling plan gene-strings of $\mathrm{O} 1$ based on the positions of the crossover point and the right crossover parts of the scheduling plan gene-strings of P2 simultaneously. If one gene number of $\mathrm{O} 1$ can be found to be the same in $\mathrm{P} 2$ based on the position sequence, replace the genes of $\mathrm{O} 1$ and P2 with 0; for example, in Figure 4, gene number ' 6 ' of the first position of $\mathrm{O} 1$ is the same as gene number ' 6 ' of the eighth position of $\mathrm{P} 2$, and ' 6 ' is replaced by ' 0 '. The remaining genes of $\mathrm{O} 1$ and $\mathrm{P} 2$ are mapped to each other in terms of the position sequence, for example, '2' to '4', ' 5 ' to '4', '5' to '4', '2' to '4' and '6' to '3' (Figure 4). Similarly, if one gene from the right parts of the scheduling plan gene-strings of $\mathrm{O} 2$ can be found in the right crossover parts of $\mathrm{P} 1$, replace the elements of $\mathrm{O} 2$ and $\mathrm{P} 1$ with 0 . The remaining elements of $\mathrm{O} 2$ and $\mathrm{P} 1$ are matched with each other in terms of the position sequence.

Step 3.5. Determine the positions of the remaining elements of $\mathrm{O} 1$ and $\mathrm{O} 2$ in the left crossover parts of the scheduling plan gene-strings of $\mathrm{O} 1$ and $\mathrm{O} 2$, respectively, and then update the elements of the positions with the matched elements of $\mathrm{O} 1$ and $\mathrm{O} 2$, respectively (see Figure 4).

SAA-based mutation operator. A mutation operator is required to generate schedules with new information after the crossover operator. Several approaches for the mutation operator, such as uniform and non-uniform mutation and immunity-based mutation operators, have been used to solve complex global optimisation problems. In this study, a new SAA-based mutation operator is designed for the algorithm. In the SAA, a temperature parameter plays a significant role in guiding and controlling the performance of the algorithm.
When the temperature reaches a high level, the SAA has a high probability of accepting a new state, while the search space becomes very small at low temperatures, and the SAA has a low probability of accepting a new state. Hence, the new mutation operator can improve the search ability and search efficiency of the algorithm by developing a novel non-linear annealing control function based on up-regulatory Hill functions in the solution space. The major process of the SAA-based mutation operator can be outlined as follows:

Step 1. Initialise SAA parameters.

Step 1.1. Obtain the initial temperature T0, the final temperature $\mathrm{T} 1$ and the current temperature $\mathrm{T}$ and set $\mathrm{T}=\mathrm{T} 0$, where $\mathrm{T} 0$ is given as: $\mathrm{T} 0=-100 \times$ $(\max ($ Objv $)-\min ($ Objv $)) / \log \mathrm{Pa}$, where $\max ($ Objv $)$ is the maximum value of the objective function, $\min (\mathrm{Obj})$ is the minimum value of the objective function and $\mathrm{Pa}$ is the initial acceptance probability.

Step 1.2. Select an initial schedule $\mathrm{S} 0$ and set the current schedule $\mathrm{S}=\mathrm{S} 0$ and the best schedule $\mathrm{S}^{*}=\mathrm{S} 0$.

Step 1.3. Calculate the function value of the current schedule $\mathrm{f}(\mathrm{S})=\mathrm{f}(\mathrm{S} 0)$.

Step 1.4. Set $\mathrm{k}=0$.

Step 2. While the stop criterion is not satisfied:

Step 2.1. Generate a feasible schedule $\mathrm{S}^{\prime}$ in the neighbourhood of the current schedule $\mathrm{S}$ using an adjacent swapping strategy.

Step 2.2. Calculate $\Delta=\left(\mathrm{f}\left(\mathrm{S}^{\prime}\right)-\mathrm{f}(\mathrm{S})\right) / \mathrm{f}(\mathrm{S}) \times 100$.

Step 2.3. If $\Delta \leqslant 0$, set $\mathrm{S}=\mathrm{S}^{\prime}, \mathrm{f}(\mathrm{S})=\mathrm{f}\left(\mathrm{S}^{\prime}\right)$, and update the best schedule $\mathrm{S}^{*}=\mathrm{S}^{\prime}$.

Step 2.4. Else, generate a random number $r \in(0,1)$; if $r<\exp (-\Delta / T)$, set $\mathrm{S}=\mathrm{S}^{\prime}, \mathrm{f}(\mathrm{S})=\mathrm{f}\left(\mathrm{S}^{\prime}\right)$, and update the best schedule $\mathrm{S}^{*}=\mathrm{S}^{\prime}$.

Step 3. Generate an annealing control function based on an up-regulatory Hill function, which can keep the hormone regulation adaptive and stable at a good accuracy and a fast speed. ${ }^{31}$ The expression is described as follows

$$
\begin{array}{ll}
\mathrm{T}(\mathrm{k}+1)=\alpha \times \mathrm{F}_{\text {down }}(\mathrm{k})-\mathrm{k} \times \Delta \mathrm{T} / \exp (\mathrm{k}) \\
\text { where } \quad \mathrm{F}_{\text {down }}(\mathrm{k})=1 /\left(1+\mathrm{k}^{\mathrm{n}}\right) \text { and } \Delta \mathrm{T}=\mathrm{T}(\mathrm{k}+1)-\mathrm{T}(\mathrm{k}) \\
\mathrm{k}=\mathrm{k}+1
\end{array}
$$

Step 4. Return the best schedule and the corresponding function value.

Step 5. Stop.

\section{Case studies}

The proposed modified GA is implemented using the MATLAB programming language. The experimental tests are performed on a personal computer with a Intel Pentium (R) with $1 \mathrm{~GB}$ memory and $3.20 \mathrm{GHz}$ processor, and the operating system is Windows XP. Two experiments are conducted: one is 
Table I. Job-related information of problem I.

\begin{tabular}{|c|c|c|c|}
\hline Job number & Process plan number & Machine number of operation sequences & Processing time \\
\hline \multirow[t]{3}{*}{ JI } & I & M9-M4-M3-M5-MI 0-M7-M I-M6 & $4-2-2-3-2-3-4-3$ \\
\hline & 2 & MI 0-MI-M2-M4-M8-M7-M3-M9-M5-M6 & $3-2-2-2-3-2-3-2-2-2$ \\
\hline & 3 & M3-MI-M2-M9-M7-M8-M4-M6-M5 & $3-3-2-2-2-2-3-3-3$ \\
\hline \multirow[t]{3}{*}{ J2 } & I & M8-M6-M9-M5-M4 & $4-5-5-4-2$ \\
\hline & 2 & M2-MI-M4-M3-M7 & $5-5-3-5-2$ \\
\hline & 3 & MIO-M3-M5-M2 & $6-5-5-4$ \\
\hline \multirow[t]{3}{*}{$\mathrm{J} 3$} & I & M3-M4-M7-M8 & $5-5-4-4$ \\
\hline & 2 & M9-M6-M5-M4 & $6-4-4-4$ \\
\hline & 3 & MI-M2-MIO-M9 & $3-5-5-5$ \\
\hline \multirow[t]{3}{*}{ J4 } & I & M8-M7-M4-M3-M9 & $4-5-3-4-4$ \\
\hline & 2 & M6-M5-M2-M8-M7 & $3-5-4-4-4$ \\
\hline & 3 & MIO-M6-M4-M9 & $5-6-4-5$ \\
\hline \multirow[t]{3}{*}{ J5 } & I & M3-M2-M7-M8-M6-M9-MIO & $3-3-2-3-2-3-3$ \\
\hline & 2 & M2-MI0-M9-M7-M5-M4-M6 & $4-2-3-3-3-2-2$ \\
\hline & 3 & MI-M9-M7-M4-M5-M6 & $4-3-3-3-2-4$ \\
\hline \multirow[t]{3}{*}{ J6 } & I & M7-M2-M5 & $6-6-5$ \\
\hline & 2 & M6-M9-MIO & 6-5-6 \\
\hline & 3 & M4-M7-M8 & $5-6-6$ \\
\hline \multirow[t]{3}{*}{ J7 } & 1 & MI-M2-M9 & $4-7-6$ \\
\hline & 2 & M3-M4-M5 & $6-6-5$ \\
\hline & 3 & M5-M7-MIO & $5-6-6$ \\
\hline \multirow[t]{3}{*}{ J8 } & I & M8-M I0-M2 & $5-6-6$ \\
\hline & 2 & M5-M7-M9 & $4-7-6$ \\
\hline & 3 & MI0-M6-M3 & $5-6-6$ \\
\hline \multirow[t]{3}{*}{9} & I & M7-M4-M9-MIO & $5-6-5-5$ \\
\hline & 2 & M3-M5-M8-M2 & $6-6-5-4$ \\
\hline & 3 & MI-M3-M5-M7 & $5-5-6-5$ \\
\hline \multirow[t]{3}{*}{ J10 } & I & M5-M3-M7 & $6-6-5$ \\
\hline & 2 & M4-MI-M9 & $6-5-6$ \\
\hline & 3 & MIO-M5-M8 & $5-6-6$ \\
\hline
\end{tabular}

Table 2. Experimental results of problem I.

\begin{tabular}{lcccc}
\hline & Dong and Sun ${ }^{32}$ & Zhu et al. ${ }^{34}$ & Mohammadi et al. $^{26}$ & Proposed algorithm \\
\hline Makespan & 30 & 29 & 28 & 27 \\
CPU time (s) & 245 & 225 & 185 & 170 \\
\hline
\end{tabular}

to show the effectiveness and performance of the proposed approach, and the other is to discuss the EIPPS model.

\section{Performance evaluation of the algorithm}

To demonstrate the performance of the proposed modified GA, two problems that were given by Dong and Sun $^{32}$ and Moon et al. ${ }^{33}$ are presented in the first experiment. The objective of this section is to minimise the total completion time (i.e. makespan). The first example includes 10 jobs and 10 machines in process planning and scheduling. Each job has three alternative process plans, and each operation of jobs can be executed on different machines. The alternative process plans and processing time are shown in Table 1. The proposed algorithm is compared with several different algorithms, including the genetic multi-objective SAA of Mohammadi et al., ${ }^{26}$ the immune GA of Dong and Sun, ${ }^{32}$ and the particle optimisation algorithm of $\mathrm{Zhu}$ et al. ${ }^{34}$ The experimental results are shown in Table 2, and one of the best schedules is illustrated with the Gantt chart, as shown in Figure 5.

As shown in Table 2, which compares the experimental results of the four algorithms, the best makespan for problem 1 is obtained by the proposed algorithm, which gives a makespan value of 27 . It is clear that the proposed algorithm outperforms those of Dong and Sun, ${ }^{32}$ Zhu et al. ${ }^{34}$ and Mohammadi et al. ${ }^{26}$ The optimal process plan is 2-2-2-2-3-2-3-1-2-2, which means that job 1 selects the first process plan, job 2 selects the second process plan and so on, and the corresponding processing times are shown in bold in Table 1. The optimal scheduling results can be described with the Gantt chart in Figure 5. The proposed modified GA is more effective than the other algorithms; its convergence iteration time is 170 , while the convergence iteration times for the other methods are 245, 225 and 185, respectively.

The work of Moon et al. ${ }^{33}$ and Mohammadi et al. ${ }^{26}$ is given as the second example to demonstrate the 


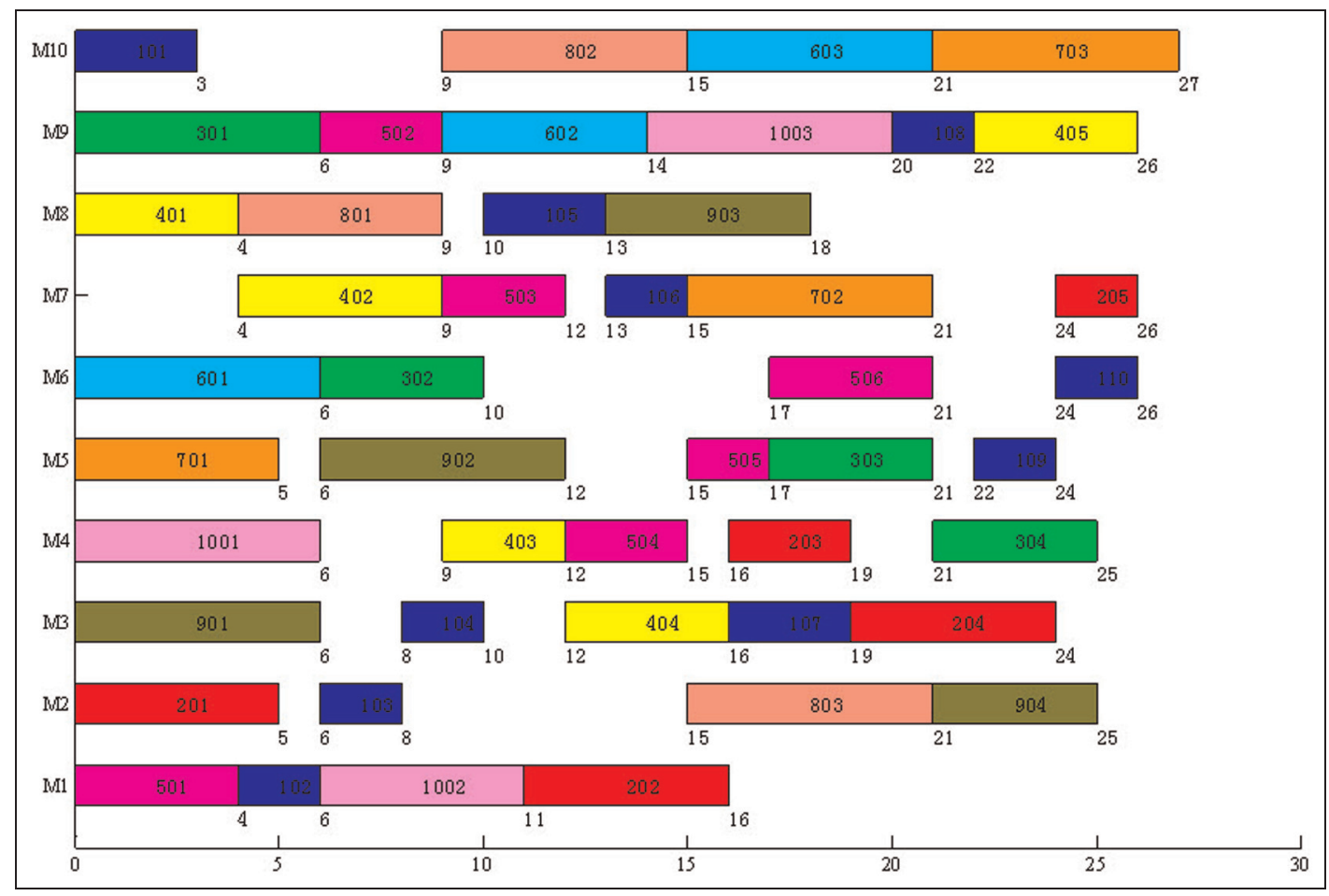

Figure 5. Gantt chart of problem I with one objective.

performance of the proposed modified GA. The problem consists of five jobs and five machines, and the makespan is set as the objective function; each job has multiple alternative process plans, and each operation of jobs can be processed on different machines. The relevant data are shown in Table 3 . Table 4 shows the experimental results obtained from the different algorithms, and the scheduling results are shown in the Gantt chart in Figure 6. The experimental results show that the best makespan is 14 and is obtained with the proposed approach, which is superior to those of Moon et al. ${ }^{33}$ and Mohammadi et al. ${ }^{26}$ The optimal process plan is 2-2-9-1-3, and the corresponding processing time is shown in bold in Table 3 .

\section{Performance evaluation of the energy-aware IPPS}

To evaluate the EIPPS model, the extended job-shop instances that involve power consumption for the machines are developed based on the problem given by Dong and Sun $^{32}$ that was presented previously. Assume that all of the machines in the study are automated, the cutting power on each machine is set to the same value, and the data for the unload power consumption of each machine can be obtained by the method developed by Liu et al., ${ }^{35}$ which is shown in Table 5. For the problem given by Dong and Sun, ${ }^{32}$ the makespan value was obtained using the optimisation approach in the experiment described above. According to the optimised plan of process planning and scheduling, the total energy consumption is calculated as 806.01. Because of the requirements for factory production, the decision-maker mainly considers the production efficiency with the objective of production time; here, the energy consumption could be reduced as much as possible, while the completion time could be delayed without affecting the delivery time, and the value of the makespan factor can be set from 0.9 to 1 . The proposed algorithm was run 10 times for the EIPPS problem. Figure 7 shows the points on the Pareto efficient frontier for the energy consumption and makespan of the bi-objective mixed integer programming model, and each point presents the average of these 10 runs.

Figure 7 describes the Pareto efficient frontier for the problem given by Dong and Sun. ${ }^{32}$ The experimental results show that a conflicting relationship exists between the energy consumption and makespan. Because the makespan is considered to be the dominant solution, average values of the makespan as short as approximately 27.5 can be obtained at the expense of high energy consumption. The corresponding average energy consumption is 813.15 . On the other hand, if a longer production time can be accepted without affecting the delivery time, the energy consumption can be reduced to 654.36 with a makespan that is increased by approximately $20 \%$. The total energy consumption can be decreased by $19.53 \%$. The Pareto efficient frontier between the energy and makespan of one run for the problem is shown in Figure 8. Note that the same makespan will correspond to different levels of energy consumption due to alternative process planning and scheduling plans, and that the minimum energy consumption can be selected while maintaining the same 
Table 3. Job-related data of problem 2.

\begin{tabular}{|c|c|c|c|}
\hline Job number & Process plan number & Machine number of operation sequences & Processing time \\
\hline \multirow[t]{2}{*}{ JI } & I & MI-M2 & $5-5$ \\
\hline & 2 & M2-M2 & 3-5 \\
\hline \multirow[t]{2}{*}{$\mathrm{J} 2$} & I & M3-M5 & $6-4$ \\
\hline & 2 & M4-M5 & $5-4$ \\
\hline \multirow[t]{12}{*}{$\mathrm{J} 3$} & 1 & M2-M3-M2 & $4-2-5$ \\
\hline & 2 & M2-M4-M2 & $4-3-5$ \\
\hline & 3 & MI-M3-M2 & $5-2-5$ \\
\hline & 4 & MI-M4-M2 & $5-3-5$ \\
\hline & 5 & M2-M2-M3 & $4-5-2$ \\
\hline & 6 & M2-M2-M4 & $4-5-3$ \\
\hline & 7 & MI-M2-M3 & $5-5-2$ \\
\hline & 8 & MI-M2-M4 & $5-5-3$ \\
\hline & 9 & M2-MI-M3 & $5-5-2$ \\
\hline & 10 & M2-MI-M4 & $5-5-3$ \\
\hline & 11 & M2-M2-M3 & $5-4-2$ \\
\hline & 12 & M2-M2-M4 & $5-4-3$ \\
\hline J4 & I & M3-M4 & 4-5 \\
\hline \multirow[t]{24}{*}{ j5 } & $\mathrm{I}$ & MI-MI-M3-M4 & $4-2-5-4$ \\
\hline & 2 & MI-M2-M3-M3 & $4-4-5-4$ \\
\hline & 3 & MI-MI-M3-M5 & $4-2-5-3$ \\
\hline & 4 & MI-M2-M3-M5 & $4-4-5-3$ \\
\hline & 5 & M2-MI-M3-M3 & $3-2-5-4$ \\
\hline & 6 & M2-MI-M3-M5 & $3-2-5-3$ \\
\hline & 7 & M2-M2-M3-M3 & $3-4-5-4$ \\
\hline & 8 & M2-M2-M3-M5 & $3-4-5-3$ \\
\hline & 9 & MI-M3-MI-M3 & $4-5-2-4$ \\
\hline & 10 & MI-M3-MI-M5 & $4-5-2-3$ \\
\hline & 11 & MI-M3-M2-M3 & $4-5-4-4$ \\
\hline & 12 & MI-M3-M2-M5 & $4-5-4-3$ \\
\hline & 13 & M2-M3-MI-M3 & $3-5-2-5$ \\
\hline & 14 & M2-M3-MI-M5 & $3-5-2-3$ \\
\hline & 15 & M2-M3-M2-M3 & $3-5-4-4$ \\
\hline & 16 & M2-M3-M2-M5 & $3-5-4-3$ \\
\hline & 17 & M3-MI-MI-M3 & $5-4-2-4$ \\
\hline & 18 & M3-MI-MI-M5 & $5-4-2-3$ \\
\hline & 19 & M3-MI-M2-M3 & $5-4-4-4$ \\
\hline & 20 & M3-MI-M2-M5 & $5-4-4-3$ \\
\hline & 21 & M3-M2-M2-M3 & $5-3-4-4$ \\
\hline & 22 & M3-M2-M2-M5 & $5-3-4-3$ \\
\hline & 23 & M3-M2-MI-M3 & $5-3-2-4$ \\
\hline & 24 & M3-M2-MI-M5 & $5-3-2-3$ \\
\hline
\end{tabular}

Table 4. Experimental results of problem 2.

\begin{tabular}{llll}
\hline & Moon et al. ${ }^{33}$ & Mohammadi et al. ${ }^{26}$ & Proposed algorithm \\
\hline Makespan & 16 & 15 & 14 \\
\hline
\end{tabular}

Table 5. Data for the unload power consumption of each machine.

\begin{tabular}{lllllllllll}
\hline & MI & M2 & M3 & M4 & M5 & M6 & M7 & M8 & M9 & M10 \\
\hline Unload power & 2.40 & 3.36 & 2.00 & 1.77 & 2.20 & 7.50 & 2.00 & 1.77 & 2.20 & 7.50 \\
\hline
\end{tabular}

production time. For example, when the makespan value reaches 28 , the associated energy consumption has minimum and maximum values of 717.74 and 784.13, respectively. Thus, the optimal objective with the minimum value of 717.74 is employed as a production plan, which indicates an $8.47 \%$ improvement in the total energy consumption. The results of the biobjective optimisation problem and the results of the 


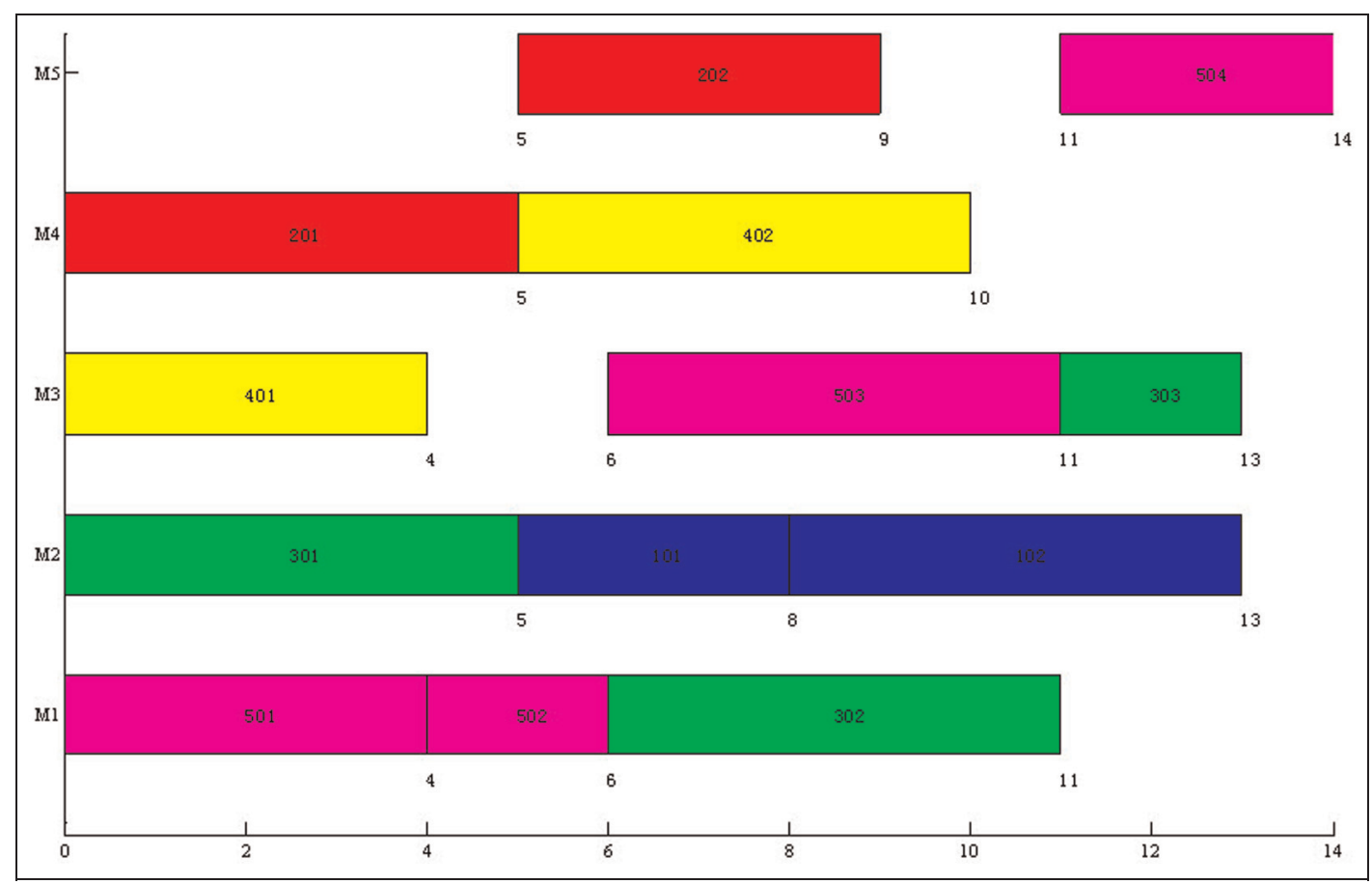

Figure 6. Gantt chart of problem 2.

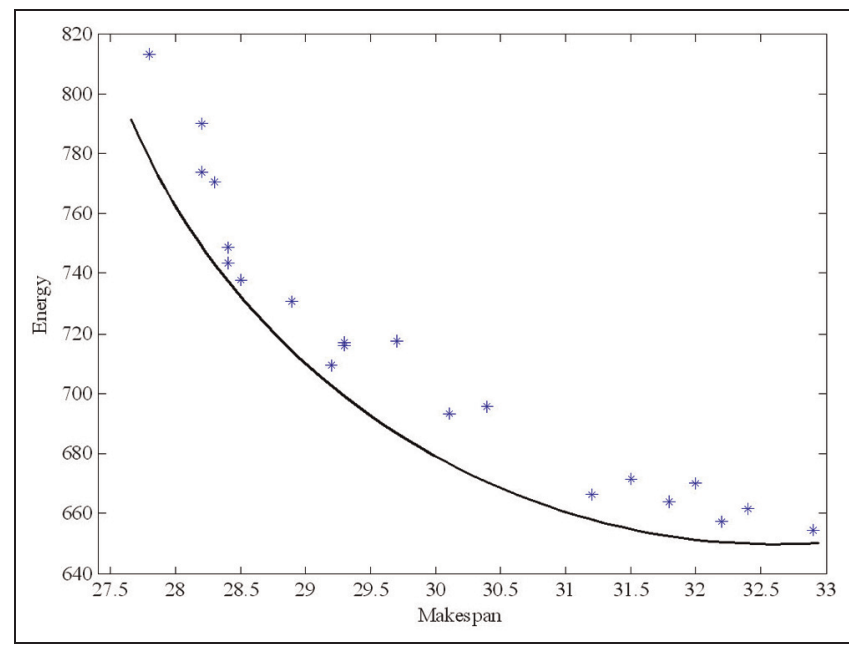

Figure 7. Plots of energy and makespan for the problem.

single-objective optimisation problem are compared in Figure 8 . In the bi-objective optimisation problem, the optimal makespan value is 27 , while the corresponding minimum energy consumption value is 785.96 . In the single-objective optimisation problem, the optimal makespan value is 27 , while the associated energy consumption value is 806.01 . The reduction of the total energy consumption can reach approximately $2.5 \%$ compared to the single-objective optimisation result. Furthermore, the optimal process plan is 1-2-2-3-3-3-11-2-1. The corresponding processing time is shown in italics in Table 1, and the Gantt chart of the scheduling results is shown in Figure 9.

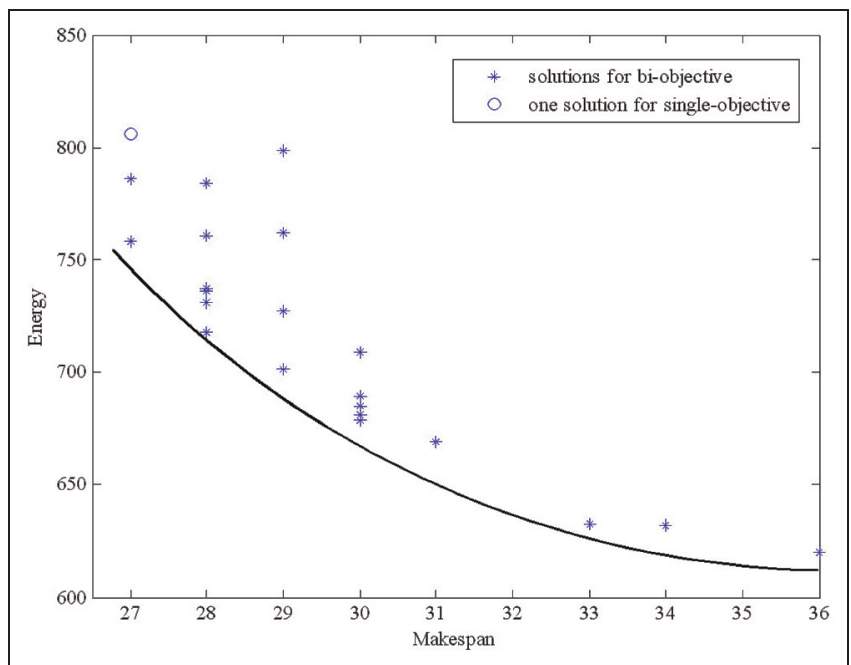

Figure 8. Pareto efficient frontier between the energy and makespan.

\section{Conclusion and future research}

In this article, we explored the energy-aware process planning and scheduling problem in sustainable manufacturing processes, and a new performance indicator of energy efficiency was considered as an optimisation objective. A model for the multi-objective total energy consumption and makespan job-shop problem was developed to describe EIPPS. To solve the multiobjective optimisation problem, a modified GA was adopted to search for the optimal solutions between the energy consumption and makespan. To verify the 


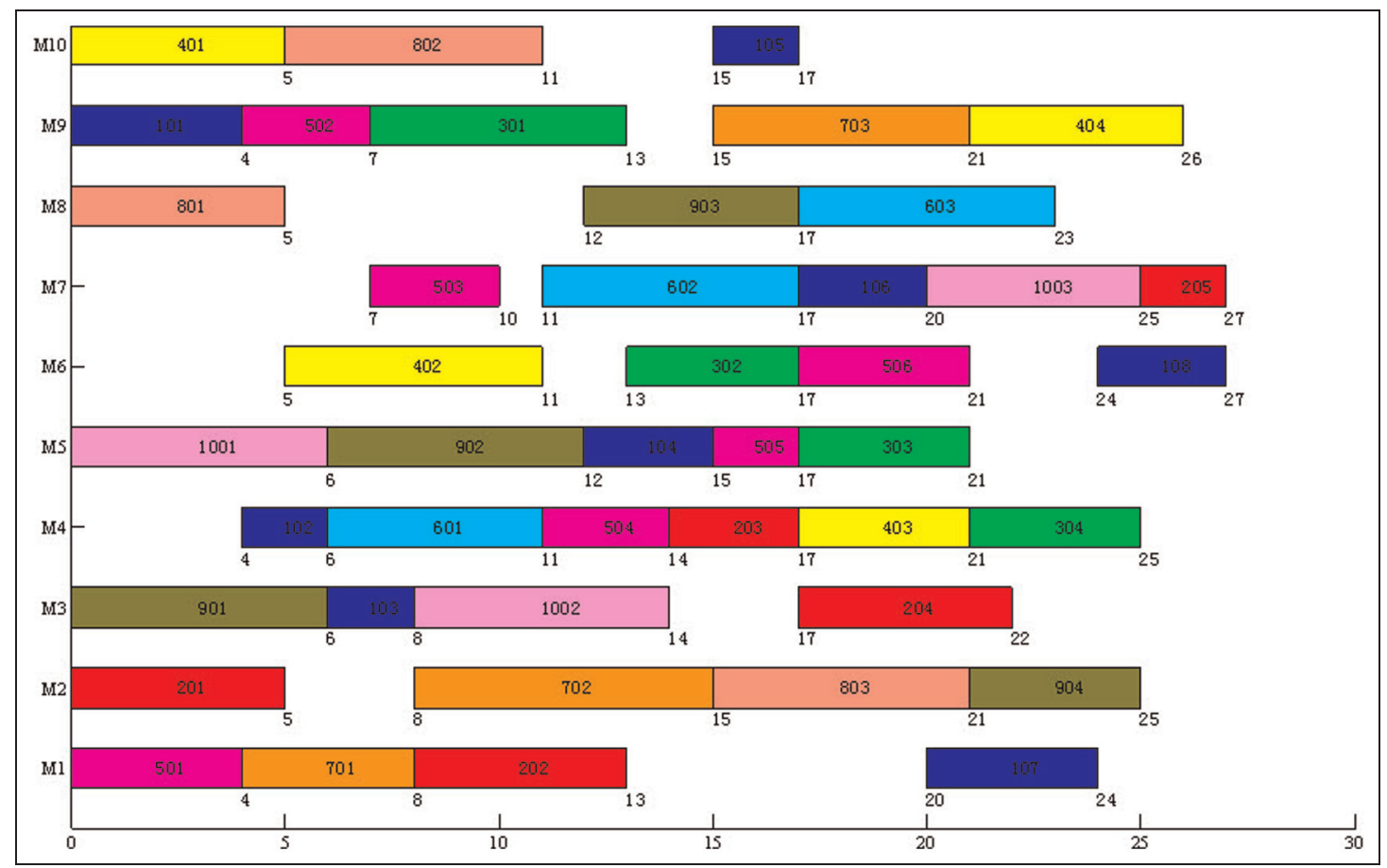

Figure 9. Gantt chart of problem I with two objectives.

performance of the proposed algorithm, a comparison experiment with other methods was presented. Biobjective optimisation problems with the objectives of minimising the energy consumption and makespan in a job shop were tested. The experimental results indicated that the proposed method can identify a set of Pareto optimal solutions in the solution space and highlighted the conflicting relationship between the energy consumption and makespan.

In conclusion, this article explored how to improve the energy efficiency of sustainable manufacturing processes by selecting proper process plans and changing the job processing sequence on machines at the process planning and scheduling levels. The decision model proposed by Mouzon et al. ${ }^{12}$ is an effective and efficient method for reducing energy consumption by turning off and then turning on idle machines. It is therefore worth studying methods of obtaining better energyefficient optimisation results in process planning and scheduling. In addition, unexpected events that may occur in real manufacturing processes, such as machine failures, rush orders and job cancellations, should be considered in energy-aware process planning and scheduling problems. Minimising the energy consumption in dynamic scheduling problems should be investigated further in the future.

\section{Declaration of conflicting interests}

The authors declare that there are no conflicts of interest.

\section{Funding}

This research was carried out as part of the CASES project, which is supported by a Marie Curie
International Research Staff Exchange Scheme Fellowship within the 7th European Community Framework Programme under grant agreement No. 294931. This research was also supported by National Science Foundation of China (No. 51175262) and the Jiangsu Province Science Foundation for Excellent Youths under Grant BK20121011.

\section{References}

1. IEA. Worldwide trends in energy use and efficiency. Paris: IEA/OECD, 2008.

2. Herrmann C, Bergmann L and Thiede S.Methodology for the design of sustainable production systems. Int $J$ Sustain Manuf 2009; 1: 376-395.

3. Duflou JR, Sutherland JW, Dornfeld D, et al. Towards energy and resource efficient manufacturing: a processes and systems approach. CIRP Ann: Manuf Techn 2012; 61: 587-609.

4. Apostolos F, Alexios P, Georgios P, et al. Energy efficiency of manufacturing processes: a critical review. In: 46th CIRP conference on manufacturing systems (CIRP CMS 2013), Setúbal, 29-30 May 2013, pp.628-633. Amsterdam: Elsevier.

5. Sheng $P$ and Srinivasan M.Multi-objective process planning in environmentally conscious manufacturing: a feature-based approach. CIRP Ann: Manuf Techn 1995; 44: 433-437.

6. Srinivasan M and Sheng P.Feature-based process planning for environmentally conscious machining. Part 1: microplanning. Robot Cim: Int Manuf 1999; 15: 257-270.

7. Srinivasan $M$ and Sheng P.Feature based process planning in environmentally conscious machining. Part 2: macroplanning. Robot Cim: Int Manuf 1999; 15: 271-281.

8. Singh S, Goodyer J and Popplewell K.Integrated environmental process planning for the design and 
manufacture of automotive components. Int $J$ Prod Res 2007; 45: 4189-4205.

9. Kai J, Zhang HC, Balasubramaniam P, et al. A multiple objective optimization model for environmental benign process planning. In: IE\&EM '09: 16th international conference on industrial engineering and engineering management, Beijing, China, 21-23 October 2009, pp.869-873. New York: IEEE.

10. Newman ST, Nassehi A, Imani-Asrai R, et al. Energy efficient process planning for $\mathrm{CNC}$ machining. CIRP $J$ Manuf Sci Technol 2012; 5: 127-136.

11. Yin $\mathrm{R}, \mathrm{Cao} \mathrm{H}, \mathrm{Li} \mathrm{H}$, et al. A process planning method for reduced carbon emissions. Int J Comp Integ M 2014; 27 $1175-1186$.

12. Mouzon G, Yildirim MB and Twomey J.Operational methods for minimization of energy consumption of manufacturing equipment. Int J Prod Res 2007; 45: 4247 4271.

13. Mouzon $G$ and Yildirim MB.A framework to minimise total energy consumption and total tardiness on a single machine. Int J Sustain Eng 2008; 1: 105-116.

14. Fang $\mathrm{K}$, Uhan $\mathrm{N}$, Zhao $\mathrm{F}$, et al. A new approach to scheduling in manufacturing for power consumption and carbon footprint reduction. J Manuf Syst 2011; 30: 234-240.

15. Bruzzone AAG, Anghinolfi D, Paolucci M, et al. Energyaware scheduling for improving manufacturing process sustainability: a mathematical model for flexible flow shops. CIRP Ann: Manuf Techn 2012; 61: 459-462.

16. Zhang L, Li X, Gao L, et al. Dynamic scheduling model in FMS by considering energy consumption and schedule efficiency. In: 2012 IEEE 16th international conference on computer supported cooperative work in design (CSCWD 2012), Wuhan, China, 23-25 May 2012, pp.719-724. New York: IEEE.

17. Liu G-S, Zhang B-X, Yang H-D, et al. A branch-andbound algorithm for minimizing the energy consumption in the PFS problem. Math Probl Eng 2013; 2013: 546810 (6 pp.).

18. Dai M, Tang D, Giret A, et al. Energy-efficient scheduling for a flexible flow shop using an improved geneticsimulated annealing algorithm. Robot Cim: Int Manuf 2013; 29: 418-429.

19. Chryssolouris G, Chan S and Cobb W.Decision making on the factory floor: an integrated approach to process planning and scheduling. Robot Cim: Int Manuf 1984; 1: 315-319.

20. Chryssolouris G, Chan S and Suh NP.An integrated approach to process planning and scheduling. CIRP Ann: Manuf Techn 1985; 34: 413-417.

21. Li WD and McMahon CA.A simulated annealing-based optimization approach for integrated process planning and scheduling. Int J Comp Integ M 2007; 20: 80-95.
22. Amin-Naseri MR and Afshari AJ.A hybrid genetic algorithm for integrated process planning and scheduling problem with precedence constraints. Int $J$ Adv Manuf Tech 2012; 59: 273-287.

23. Morad $\mathrm{N}$ and Zalzala A.Genetic algorithms in integrated process planning and scheduling. J Intell Manuf 1999; 10: 169-179.

24. Guo YW, Li WD, Mileham AR, et al. Applications of particle swarm optimisation in integrated process planning and scheduling. Robot Cim: Int Manuf 2009; 25: 280-288.

25. Li X, Gao L, Shao X, et al. Mathematical modeling and evolutionary algorithm-based approach for integrated process planning and scheduling. Comput Oper Res 2010; 37: 656-667.

26. Mohammadi G, Karampourhaghghi A and Samaei F.A multi-objective optimisation model to integrating flexible process planning and scheduling based on hybrid multiobjective simulated annealing. Int J Prod Res 2012; 50: 5063-5076.

27. Liu F, Xu Z-J and Dan B. Energy performance of machining systems with its application. Beijing, China: China Machine Press, 1995.

28. Khoshnevis B and Chen Q.Integration of process planning and scheduling functions. J Intell Manuf 1991; 2: $165-175$.

29. Holland JH. Adaptation in natural and artificial systems: an introductory analysis with applications to biology, control, and artificial intelligence. Cambridge, MA: The MIT Press, 1992.

30. Kirkpatrick S, Gelatt CD and Vecchi MP.Optimization by simulated annealing. Science 1983; 220: 671-680.

31. Rene Thomas and Richard D'Ari. Biological Feedback. Boca Raton, Florida: CRC Press, 1990.

32. Dong CY and Sun SD.Immune genetic algorithm job scheduling process and collaborative optimization. Mech Sci Technol 2007; 26: 761-766.

33. Moon $\mathrm{C}$, Lee $\mathrm{YH}$, Jeong $\mathrm{CS}$, et al. Integrated process planning and scheduling in a supply chain. Comput Ind Eng 2008; 54: 1048-1061.

34. Zhu H, Ye W and Bei G. A particle swarm optimization for integrated process planning and scheduling. In: 2009 IEEE 10th international conference on computer-aided industrial design and conceptual design: e-business, creative design, manufacturing (CAID and CD'2009), Wenzhou, China, 26-29 November 2009, pp.1070-1074. New York: IEEE.

35. Liu $\mathrm{Y}$, Dong $\mathrm{H}$, Lohse $\mathrm{N}$, et al. An investigation into minimising total energy consumption and total weighted tardiness in job shops. J Clean Prod 2014; 65: 87-96. 